\title{
Note ON UsAge
}

\section{Regions and Regional Wine}

I follow the scholarly custom of capitalizing the names of wine regions and use lowercase for the wines that originate from these regions. Hence, Burgundy, Bordeaux, and Champagne refer to the regions, whereas burgundy, bordeaux, and champagne refer to wines from these regions.

\section{Metric and American Equivalent}

\author{
1 hectare $=2.47$ acres \\ 1 liter $=0.26$ gallons \\ 1 hectoliter $=26.42$ gallons
}

